\title{
ARCHITECTURAL LARGE CONSTRUCTED ENVIRONMENT. MODELING AND R.T. INTERACTION USING DYNAMIC SIMULATIONS
}

\author{
P. Fiamma ${ }^{\text {a }}$ \\ ${ }^{a}$ Civil Engineering Department, University of Pisa, Italy \\ paolo.fiamma@ing.unipi.it
}

KEY WORDS: simulation, architectural design, dynamic interaction, understanding architecture, "Spatial Analysis System"

\begin{abstract}
:
How to use for the architectural design, the simulation coming from a large size data model? The topic is related to the phase coming usually after the acquisition of the data, during the construction of the model and especially after, when designers must have an interaction with the simulation, in order to develop and verify their idea. In the case of study, the concept of interaction includes the concept of real time "flows". The work develops contents and results that can be part of the large debate about the current connection between "architecture" and "movement". The focus of the work, is to realize a collaborative and participative virtual environment on which different specialist actors, client and final users can share knowledge, targets and constraints to better gain the aimed result. The goal is to have used a dynamic micro simulation digital resource that allows all the actors to explore the model in powerful and realistic way and to have a new type of interaction in a complex architectural scenario. On the one hand, the work represents a base of knowledge that can be implemented more and more; on the other hand the work represents a dealt to understand the large constructed architecture simulation as a way of life, a way of being in time and space. The architectural design before, and the architectural fact after, both happen in a sort of "Spatial Analysis System". The way is open to offer to this "system", knowledge and theories, that can support architectural design work for every application and scale. We think that the presented work represents a dealt to understand the large constructed architecture simulation as a way of life, a way of being in time and space. Architecture like a spatial configuration, that can be reconfigurable too through designing.
\end{abstract}

\section{INTRODUCTION}

\subsection{Topic of interest}

This work is made from the architectural background, and aims to be a research contribute inside the technical and cultural "gap" between the needs of designers and the needs of the endusers, both involved in the interaction with the simulation.

How to built and to manage a large size architectural environment able to be a knowledge base of different actors, involved in the design and decision process?

In our experience, the "flexibility" of the interaction with our 3D simulations. becomes every day more important.

We can affirm that, in the current needs of architectural designing, how to have interaction with the model is so important how to build it. At today, it is more and more necessary to identify procedure and techniques to communicate and share the design concepts, often making use of new technologies and searching for the most suitable instruments which can best enhance a dialogue between actors of the design process and their audience.

From a scientific point of view, the goal is how to manage the data exchange between designers and information models support (Eastman C. 2009).

In addition, how becomes possible to increase the efficiency of the design process whenever each actor shares with all the others a part of the knowledge deployed in the project.

For the designing activity, it is necessary a sort of "balance" between how building the model and how using it, especially if we want to use large digital re-construction of environment. The 3D interaction issue for large architectural sites, is part of this balance. In this crossroad between the designer activity and the digital technologies, we know how it is important the activity of software-programming (Kobayashi Y., 2008), that allows the designers to make flexible the digital resource according with their specific and various needs.

\section{2 "Complex" architecture as architecture on a large scale}

What can be considered a sample of "complex" architecture? Obviously there are several possible answers. Probably we can reduce the number of the possibilities, thinking who should have interaction with this complexity.

So, a complex architecture could be a building façade for who must work about it, ... we can have a complex scenario of internal degradation inside a complex building for who must renovate and reuse that building, ...we can have a complex architectural distribution of volumes on a large scale, for who must re-design and re-use a part of the city... a complex architecture can be a street scenario too (i.e. an old boulevard). Engineers and architects, are useful to understand the urban scale like a complex architecture: urban scale can be a "ensemble" of architectural complex aggregations.

In fact, the architecture in the urban context, has several meanings and targets, changing continuously. Buildings can be a new "horizon" for people moving or represent a develop of the streets, intercepting the life flows of the town. In addition, the shape and the constructive decision can represent extensions of urban space: i.e. square and building covers.

To sum up, the architectural large environments are a real "system of connections" between people, landscape and constructions.

Therefore, we know very well which are the problem to use the final result of large scale architectural simulations: the 3D model becomes, often, to much "heavy" and no easy to managed, especially for a "normal" use.

The presented paper is oriented to the needs of the final users too. Please note, that, in the case of study, the end-users group 
is composed by a different actors of the design process included no-technical actors too.

\section{THE CASE OF STUDY}

Our case of study regards how offers a common knowledge base for actors involved in the public decision about, generally speaking, our town as a complex built environment.

So the idea was to try a realize a simulations, of a large architectural environment, able to become a base for different applications (about i.e. the design and re-construction of some buildings or entire blocks of the town...).

The project is really complex, and in progress, but the first phase is already made. We present our procedural method used for modeling and navigate a large 3D virtual constructed environment: the town of Pisa.

\subsection{The construction of the architectural environment}

We think that an old city environment is a cultural heritage, so present in the normal way of thinking the local life, especially by the local people. Therefore we wanted to realize a technical work but with attention to the significant of the social context.

Our work is a step to some commons goals:

- to recreate the environment, "translating" in the 3D simulation, the real perception of the constructed environment by the people;

- to realize a interaction with the virtual environment, using a "dynamic mode": the same that the inhabits have "with" their town. One idea in fact, is "feeling" the interaction with an archeological site; a total different idea is "feeling" the interaction into an known "urban life" environment (Fukuda T. 2007);

- to use a very large size model and a new "flexible" real time interaction, without to need sophisticated resources: one of the goal was to set up an "executive" solution in order to offer to everyone the possibility to use the result of our work, using the commercial digital hardware and software they already have or can easy buy.

The focus is how optimize, in a limited time working, the input coming from my Municipality and how to transform it in an accessible knowledge for different actors of the design process that will have interaction working together on the simulation, but that, usually, comes from really different cultural and technical background (designers, tech-people of the public Administration, citizens, politicians..).

So, it was not possible, i.e., to work in order to find algorithms for generating consistent 3D models from a combination of data sources... first, because the time was not sufficient to have a performing solution, and, second, because was impossible to include computer scientists in the procedure.

\subsection{Procedural method for modelling}

About the construction of the model the technical problems were:

- how to create a 3D model of a so large historical urban context?

- how to maintain the impact of the historical aspects, such the "grammar" of the ancient streets, the skyline of the ancient town, where the big size of the most important monuments emerges?

- how to create a different cultural topics under the same technical vision and possibility of reading?
- how to create a model that can mix all together these aspects without generating a sort of confusion for the users, that can be not accustomed to seeing an dynamic urban scenario?

In order to "put together" several inputs from different sources and to have a final model, running in the same digital platform, there are, usually, two different ways: or to import in an only software the different data files from the others software and making the 3D model and the phase of the interaction, inside the same final digital environment; or to use a "strata" of software, able to realize, step by step, the complete procedure: modelling the simulation, visualizing the results and having interaction with it. With respect to the features that the final modality of interaction must offer, we have chosen the second way.

This decision allows to maintain the process "architectural design oriented", avoiding some frequent programming problems, that happens during the phase of the set up of the software environment. In addition, on this way, it was possible to optimize the interface between the different software, usually used by every single actor. During the work, the first difficult, was to import the data due to the different quality level of the input files. After the importing phase of the shape file in the navigation software, the result was a very large size file, and was really impossible to manage it, using the common hardware and software devices and solutions.

In order to resolve the problem, we have made several tests and used different procedural options, but the result was always the same. To resolve definitely, the problem (Figure 1), we have analysed deeply the file that we wanted import and we had observed a plethora of "strange" shapes that were not real buildings but a sort of a presence of little construction, shorter than 2 meters.

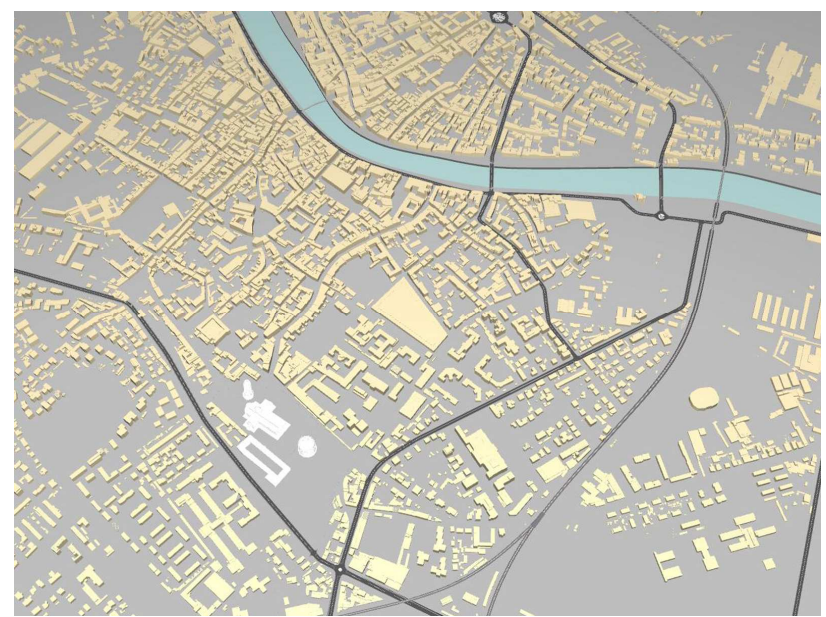

Figure 1. One step of the architectural model

A successive analysis of the situation has not found any correspondence between these shapes and significant architectural objects. Probably, these data (included in the file that we have received) were the result of an acquisition from a mix of sources, uncritically acquired: aerial photography, direct observation, data imported from some wrong data base.

From our point of view, these type of data, were real useless. According with our needs, to have correct elements, deleting this plethora of data, has incredibly reduced the size of the work in progress file.

A second difficult was to transform this file in a base for the successive final 3D model: in fact, several spatial geometries and volumes of the buildings were not "closed". To overcome 
this step, the only possibility was to export the file in a conventional software and to complete or to rebuild that geometries.

In these phase was necessary to clean the files from possible residual texture, or superficial treatments, in order to set up the next phase: to have a sort of hierarchical order, related to the "function" of the real buildings according with the colour of the surfaces (historical centre, develop zone, architectural emergences, ... Figure 2)

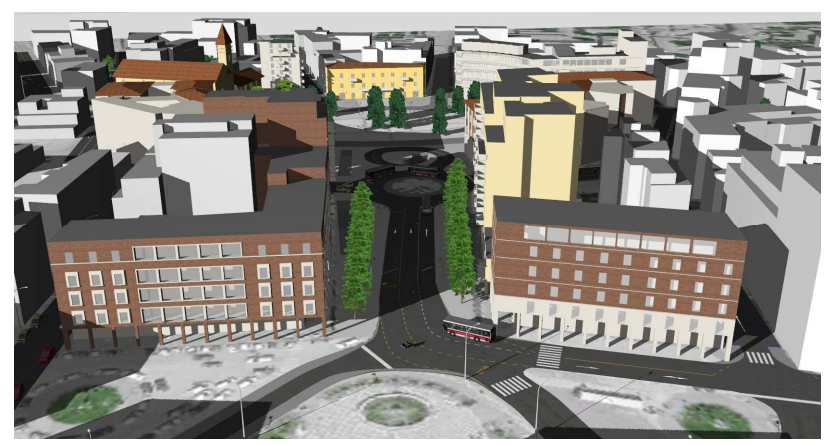

Figure 2. Different treatment of the surfaces of the buildings

Therefore, in the third step specific textures on some specific buildings have been put, in order to visualize some important difference between the function of the buildings..

To obtain a readable final impact, due to the size of the model, we have chosen to mix the ongoing results with a typical representation of the architectural environment: the so called "plan-volumetric-definition" (Figure 3).

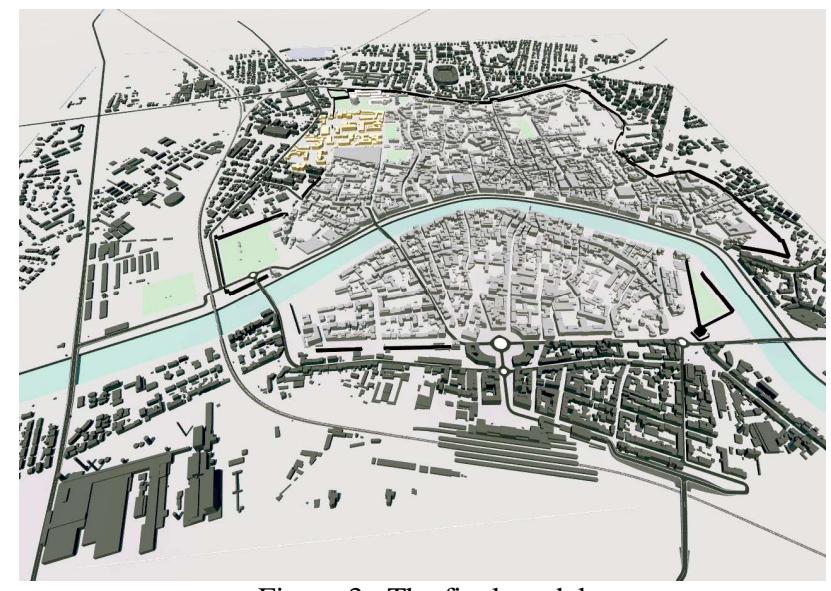

Figure 3. The final model

Please note that, from a computer graphics point of view, the problem of the visualization of the incoming volumes, in a dynamic scenario, is one of the more complex topic and it is really difficult to be resolved (objects in the virtual environment, appear "suddenly": that does not happen in the real word).

But this is a problem for computer scientists... in order to have a more realistic models; vice-versa, from our point of view, this topic makes no sense. In addition can offer more difficult to some end-users, to understand what is happening during the interaction phase with our simulation. Therefore, we have indicated only some detailed façades for some specific buildings, that are significant in the model.

It is not just a problem of time and of resources. Obviously, we know that a photorealistic simulation needs a lot of time: i.e., to make digital photographs, or to scan the buildings, or to generate video sequences, in order to have texture images for photo-realistic texture mapping. But, in same cases is not fruitful for architectural designers to use three-dimensional photo-realistic models to take decisions and to develop their ideas.

\subsection{Which kind of interaction for the architectural simulation?}

Generally speaking, we know that, one of the most important problem about the use of the simulations, is how to "extract" no only data, but information from our models.

On the one hand, the simulations become more and more powerful, thanks to the power of the computation devices, but, on the other hand, becomes more and more necessary to have new efficient modalities of interaction with that simulations. That is the focus: the concept of "interaction" is different, according to the different disciplines that use the simulation process.

During the history of the Architecture, the topic of "interaction" has had, always, several new and deep meanings, following to the trend and the design needs. In addition, in the current phase of the architecture is deeply changing the meaning of concepts like "model" and "simulation" too.

I prefer to speak about "simulations" and not only about "model", because the concept of the model is, nowadays, more related, in the design field, to the media/arts culture: the "electronic" model. This model is a sort of "pure" representation, that, during the design process, is made before or after the architectural idea, the design concept... on the contrary, the meaning of the simulation is something that happens during the architectural idea; the simulations is "inside" the design process ... it is something that can verify the idea of the designers. To understand this difference, it is necessary to think at the newest design digital methodologies like the Building Information Modelling (B.I.M.), the Parametric Design, the Generative Design. For these methodologies the simulation of the design is not a single product but a "process". Nowadays, the idea of the architectural design itself is becoming a process... We wanted to offer to the users a interaction of the model that can allow the simulation of the real life, happening in the architectural environment.

To sum up:

- how to have a real time interaction that can help designers to understand this kind and so large model?

- how to have a flexible change of the scale, from the ancient part of the town, to the new one?

- how to offers for the users a immediate understanding of the different flows (vehicles, busses, pedestrian...) inside the model?

- how to combine in a flexible manner, the real time visualization with the dynamic interaction between the objects inside the 3D model?

\section{SPECIFIC SOLUTIONS FOR SPECIFIC NEEDS}

\section{1 "Power" of the art}

In different situations, I have used the "game engine" software. This technology is, at the state of the art, one of the most powerful approach to have dynamic interaction with the simulations.

From my research partnership with the Georgia Technology Institute of Atlanta, I know well the performance in terms of flexibility and interactivity of the game engine resources, and, in addition, how it is possible to use these resources in different 
scales (from a single building to an entire town). In the following pictures there are some frames from movies recorded from interactive applications, implemented using Unreal Game Engine. Two samples of simulation with "medium" polygon version: buildings of the Georgia Tech Campus (Figure 4) and the Peachtree Corridor (Figure 5).
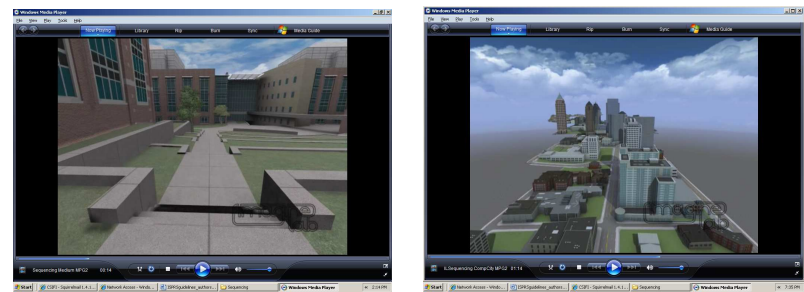

Figures 4-5. Sample of medium polygon version

Such a comparison with the presented paper, it is the impressive extensive model of the Peachtree Corridor, one of the most important way of the American city of Atlanta: more then $20 \mathrm{~km}$ long. Please note, that Peachtree Corridor is a "low" polygon model, which means that geometry is simple attention how buildings are meeting the ground: as a result in the game version, users can not go any lower then 25 meters. Building heights and locations are fairly accurate.

Another difference was the level of the involved resources: for that project, the research-professional group ordered 2 airplane and one helicopter flights. One collected LIDAR data, another took hi-res vertical aerial photography $(1$ pixel $=15 \mathrm{~cm})$ and helicopter took over 800 hi-res, oblique angle photos as a reference for texture creation.

The size of model push the interaction to be dynamic mode oriented, but only in one direction. So the architectural scale could be lost. On the contrary, we are useful to understand the architectural dimension, in the archaeological field. In the Figure 6 a sample of a possible interaction with the archaeological model, but conceived from the point of view of the designer's needs.

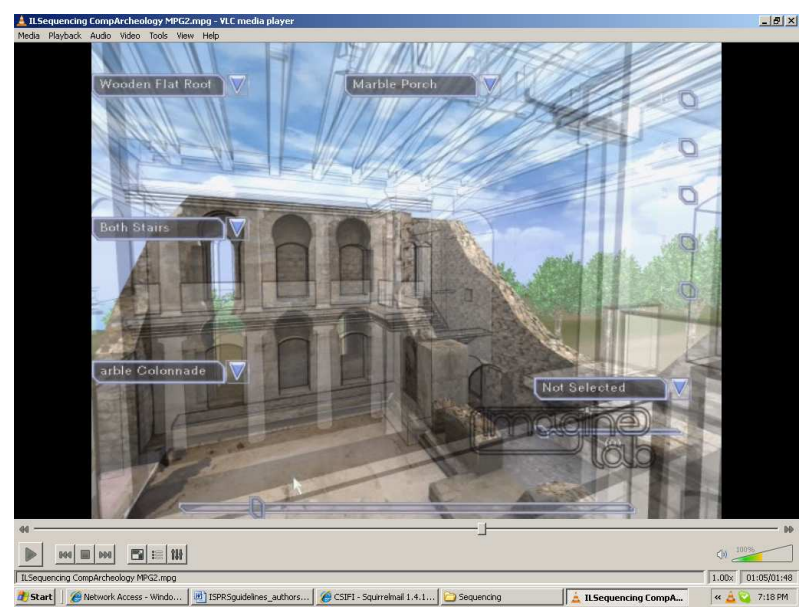

Figure 6. Game engine application

In the sample, what we consider very important, is not just the visualization features (even if really power), but the possibility to use the digital resource inside the design concept, i.e. to help designers to compare different solutions at the same time. In the picture, how to show interactively different ideas about reconstruction of the building, is showed. With different commands and controls (buttons, sliders, data base, etc.) two software strata can realize an interesting tool to show, in real time, different hypothesis of reconstruction that implied materials, technical components, costs, constructive solutions (Figure 7).

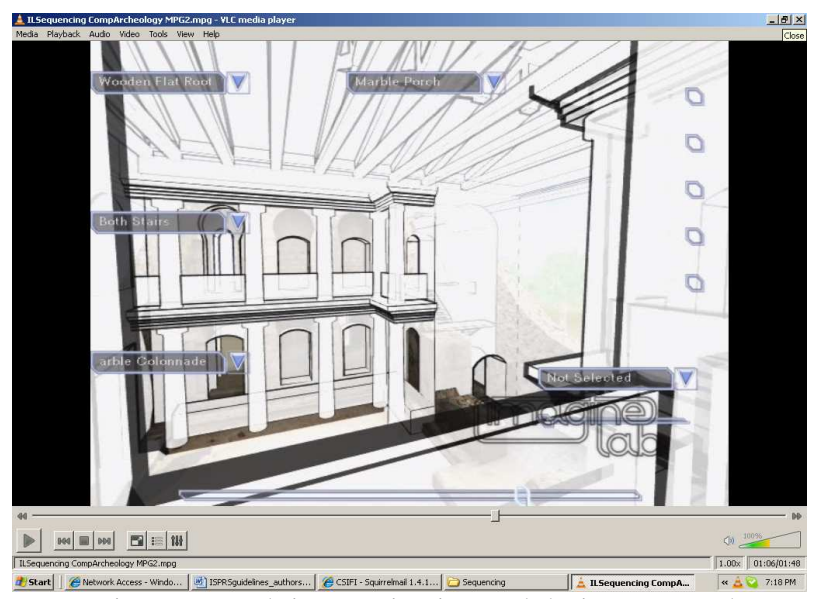

Figure 7. Real time navigation and design proposal

\subsection{Possible added value}

To sum up, game engine techniques are necessary to built and to show large size architectural models included an high levels of detailed textures in order to have a very realistic output (Lesniewski T. 2007). But in the present case of study I have decided to try new resources in order to have some added value. In fact, in our work, the look of the 3D model was made, to overcoming the normal difficult about the realistic approach.

Therefore, because the final interaction was not visualizationoriented, the use of the game engine approach was fine from a theoretical approach only. Architects are already useful to visualize architectural models using the common walkthroughs, fly paths, and others common options. On the contrary, we had need to introduce something to obtain a more analytic series of path: a interaction, programmed but "flexible" as well, such the normal uses of the flows of our towns. We wanted to have an interaction with the simulation, in order to evaluate the architectural constructions from the technical point of view but, in the same time, in order to offer to the end-users the opportunity to feel the relationship between the space and the events happening in it.

For our needs was necessary:

- to have a more realistic approach of the use of the constructed environment;

- to recreate a interaction with the model, by the point of view of the citizens, choosing, every time, streets, squares, specific locations (natural ground, street views, building levels);

to introduce a dynamic elements like traffic and pedestrian flows, on analytic bases;

to analyse a really high number of multiple positions (by pedestrian and traffic flows).

\section{FLOWS GENERATOR}

\subsection{Added value for architectural design evaluation}

If the focus is not just to "explore' the simulation in a dynamic way, but to" transfer" in the simulation the normal dynamic of the life, changing during the interaction process, in a interactive manner, the only way was try a new approach...

There are some types of digital resources coming from the research about the dynamic micro-simulations in the built 
environment. Using this software is it possible to simulate pedestrian or traffic flows. I think that, one of the added value, is the possibility to have, simultaneously, real time and script... and, in addition, subjective or from outside point of view.

These methodologies to approach the built environment offer the opportunities to realize flows of cars, people, "objects".. therefore, the interaction with the simulation can happen in a dynamic scenario, and by a in motion point of view.

There are some new research about the so called "architectural in motion", that offer a interesting background in literature. Generally speaking, the built environment is normally understanding by people in motion.

In addition is true that a large architectural model implies a sort of "in motion interactive dimension" to be, definitely, understood, according to what happens in the real life. This dynamics can happen for a single building too, if evaluated like a part of a built "context" (a system..).

On the one hand, there are technical needs: accessibility, use, functions, safety, materials; on the other hand there are needs of the perception: distances, spaces, connections, proportion, impact. The first idea of every plan, must be verified, from a several point of view, in order to become "design". Since some decades the architectural "opera" is becoming no more static but dynamic, as well.

\subsection{Integration of resources}

I have chosen a micro-dynamic simulation solution that I am developing in partnership with a several international research centres: "UcWin Road'.

Understanding the current design trend, it looks like very interesting, to observe the "crossroad" of digital approaches between architecture and geo-references. Goggle team, i.e., is developing "Sketch Up" software in order to model 3D buildings more and more efficiently, starting from the Goggle Earth base.

Vice-versa, in the professional activity, some architectural works use already geo-referencing techniques: i.e. in order to evaluate the environmental impact of constructions.

Because our case of study is a common base for multiple application, our decision was to test a digital resource able to combine:

GE: Google Earth application: to reference in the physical space the simulation of the large size architectural environment (Figure 8).

GIS: to import "shape files" into the software (Figure 9).

3D: to evaluate different architectural design and restorations of some parts of the real town

DMS Dynamic Micro Simulation flows generation: to have specific interaction with the simulation

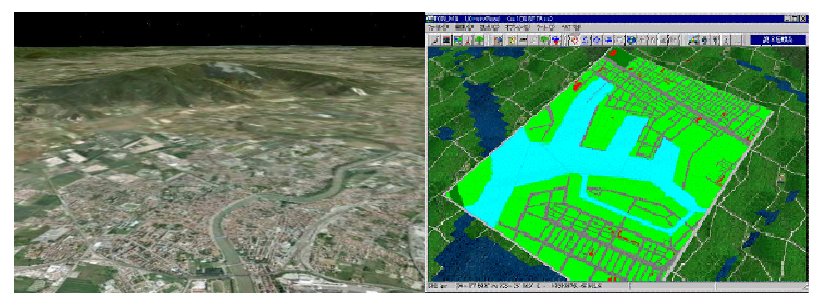

Figure 8-9. Google Earth + GIS application

\subsection{Interaction by flows}

If we think to the object observing and the subject observed, the nature of the simulation and of the point of view: both static or dynamic, the presence or not of the real time interaction...we will have almost around 20 different typologies of interaction (extract data) .

We have generated all the existing space using by citizens, overlapped by the people flows (Figure 10).

We have calculated a very high number of positions and paths, that we can use directly in a subjective mode, or as locations for the dynamic citizen flows.

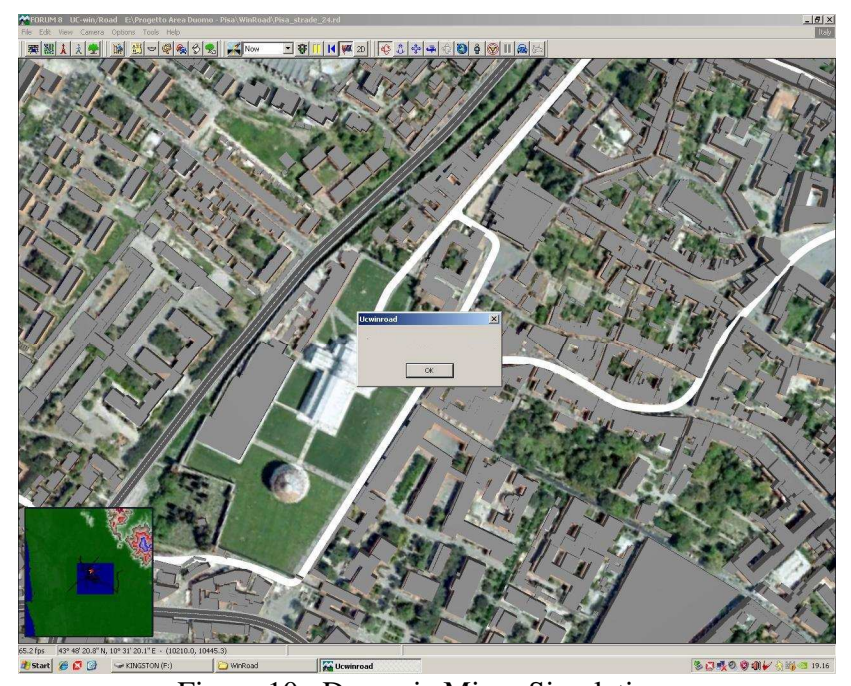

Figure 10. Dynamic Micro Simulation

Please note that all these spaces are related each other, also, we can have single space or group of spaces, usable for real time interaction. In addition, we can have a real time interaction with the architectural simulations, while we are surrounding by a plethora of dynamics programmable flows of people and vehicles.

Every variable is based on analytic computational support, and specific algorithms generate the flows, according with the real happenings. It is possible to manage the system using different approaches: scheduled format, analytic procedures, real time 3D output... Is interesting thinking how some usual paths (sky view or flight of bird) are no more necessary: that can be used just to shift more efficiently, i.e., the choice of the path for the real time interaction (Figure 11).

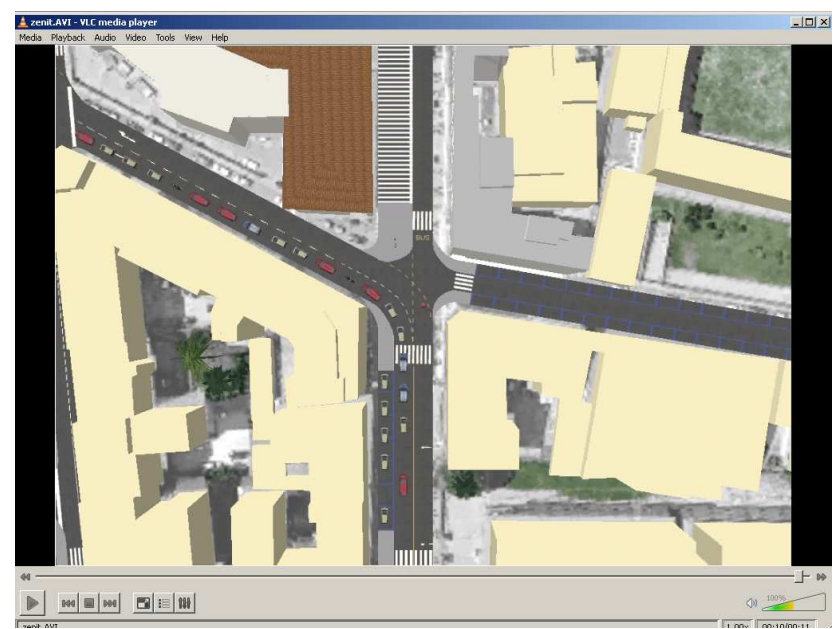

Figure 11. Traffic flows

Because the focus is to introduce the dimension of dynamic connections to evaluate the large architectural model, it is necessary to have a completely programmable device and to program scripts as well. In our case the procedure to manage the 
interaction with the simulation, is more important than the details level of the model (that we had to overcome but that we can upgrade if it is necessary). In the final simulation, every single building or architectural complex can be evaluated from hundreds of real positions, static or dynamic, while it is possible to change scenario: shifting current situation or design situation (Figure 12), in order to have immediately, i.e., distance, proportions, accessibilities, visual angles, tolerances... What we realize is a mix of interactively and iteratively procedure to conceive, to understand, to change, to design and to share design architecture.

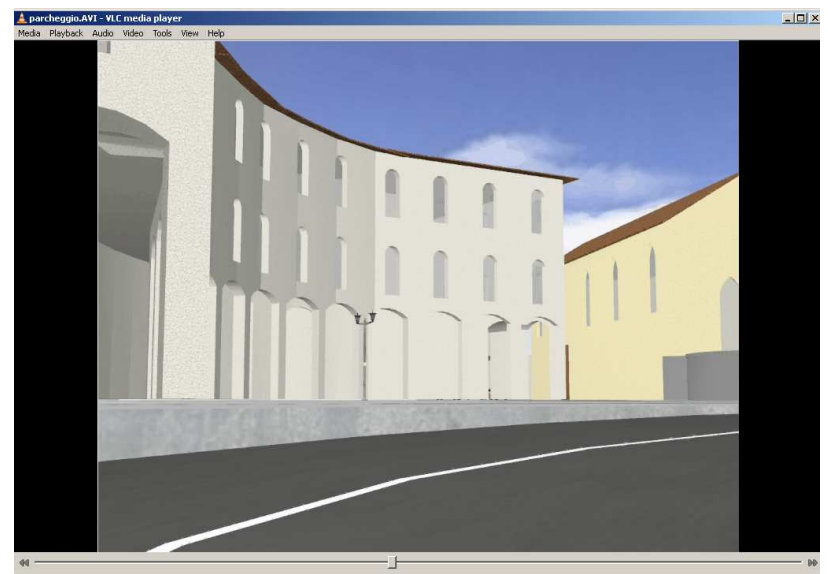

Figure 12. Sample of view from the street level

Thanks to the moving, this type of interaction, helps actors of the design process, to re-discover, one of the more important concept in the evaluation of architectural scenario: the "full and the empty" binomial, as genetic factor of the design idea.

Please note, that architecture is not only a backstage: the integration between human flows and architectural spaces is a "connection" (i.e., the constructive decision about the shapes and the materials can represent extensions of the urban space, like square and building covers).

To sum up, a project is not the object in the site but rather it is the field of relations that designers intend to inject in the context: a field of relation that has a certain range to include the surrounding of their intervention.

The architectures in the urban context can have several meanings: can be new "horizon" for people moving or become a develop of the streets intercepting the life flows of the town. The real time visualization, the "pre-scheduled-real time" visualization, the sequence of flexible pre-scheduled static points of view, can be used in a different ways using scripts too. On the one hand we can evaluate the dynamic movements that happens inside the virtual environment. On the other hand it is possible to improve the interaction with the architectural environment (i.e. looking around from inside the flows), or changing the speed and the time of the interaction mode itself (Figure 13).

An architectural complex system is definitely something that is strong related with its use by the citizens. Several technical decision must be people-oriented: on the one hand because the current evolution of the urban architecture is to extend the pedestrian zone, on the other hand because, designing architecture, it is necessary to conceive technical solutions that can preserve the quality of the life and the safety of the endusers. Both concept converge in public buildings built to extend the in-door/out-door use. Nowadays, there are several in-door situations (complex building as the modern Mall or the big airport) or out-door situation (like the renovation of old parts of the towns) where the flows of people, of stuff, of devices, are really part of the architectural design ideas, connecting public use and architectural constructions.

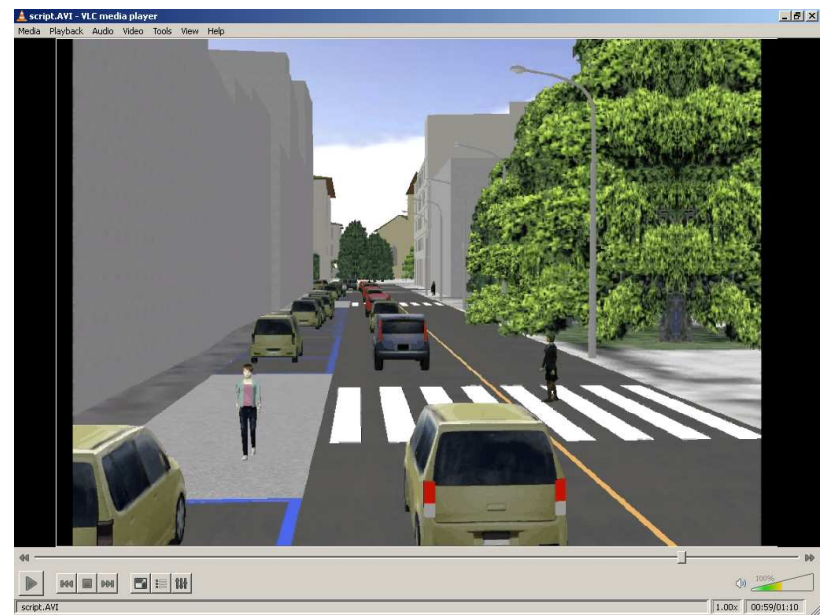

Figure 13. Flows people interaction in the architectural space

It is a propriety of the Architecture that we usually forgot: the historical architectural built environment were born to aims this focus: connecting people.

To generate pedestrian flows is a way to evaluate several technical solutions that our architecture must have, from a internal and external use (Figure 14).

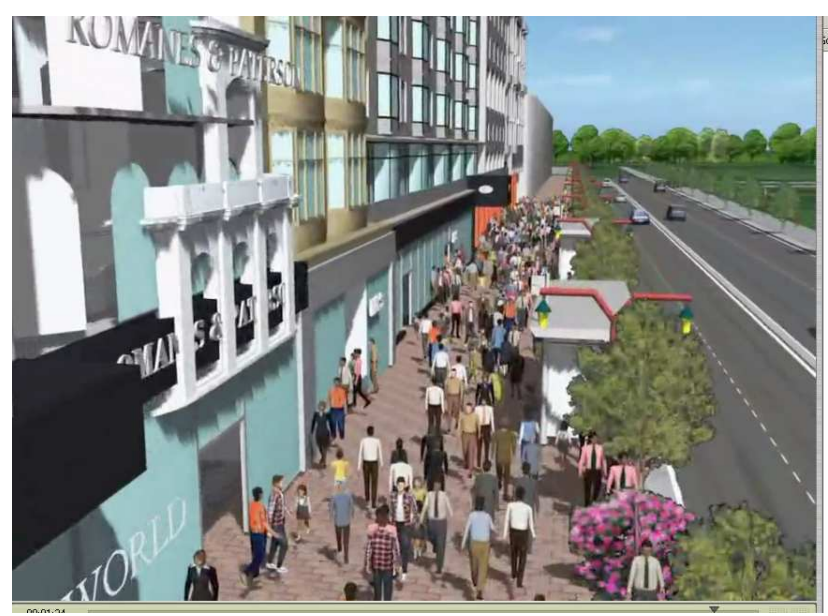

Figure 14. Sample of pedestrian simulation flows

\section{RESULT}

The presented simulation can represent a base of knowledge that can be implemented more and more. Please note, the visualization is only an output. We have shown the simulation to different audience and the result is very impressive in both situations, recorded or real time: the dynamics of the interaction emerges like a new resource to understand the architectural environment. Also, all the people invited to see the simulation, receive a big impact and discover the new facilities for the design in the virtual reconstruction thanks the methodology and the software that we have used.

From our point of view, is very interesting to observe, users of the simulation understanding the virtual environment like "own", because the model offer a very realistic impact even if the majority of the time, people is walking or driving between geometrical (and no-photorealistic) shapes. 
Obviously, tech-people can understand distances, spaces, volumes, constructive elements, and the impact to the built environment of the designing architecture and future constructions: therefore, they can extract information useful for working.

The following projects will use the prior like a knowledge base and the model can be part of a work in progress large data base, where several technical information will be linked to every single building.

In addition the model is very useful to obtain a complete understanding about the connections between pedestrian flows, public spaces and public buildings. We think that the presented work represents a dealt to understand the large constructed architecture simulation as a way of life, a way of being in time and space. Architecture like a spatial configuration, that can be reconfigurable too through designing.

\section{CONCLUSION}

\subsection{About the construction of the simulation...}

If we consider the total procedure: we have had to overcome three different steps:

- to import the different data sources from their original format (shapes file from the Municipality, 3D monuments models proprieties of the Opera del Duomo, our h/s devices)

- to use different software resources (GIS, CAD, 3D modelling, dynamic micro simulation )

- to have interaction with the simulations using real time, script, and scheduled paths.

To sum up, the research has confirmed one of the biggest problem in such type of works: the interoperability between the different software resources.

\subsection{About the procedure and the methodology ...}

How can we use a large scale 3D model in the field of architecture? If the way is the interaction: how can we use this interaction? It is already know what is happening in the architectural field about the traditional using of the $3 \mathrm{~d}$ models for architectural design: models are isolated and become finished objects, closed within themselves.

From a theoretical point of view, the current meaning of the model is something related to the simulation of the performances of the real object: they must be related to the building that they will constitute; in fact, they can not have only value as icons; they must have a value like simulation.

Into an architectural environment, especially on a large (urban) scale, the simulation should recreate the conditions to use the environment itself. As people, we live surrounding by the environment at different levels.

Therefore, one of the most important aspect in the simulation, can become how to recreate the possibilities in order to using the simulated environment. In different words: we are approaching the problem of "exhibiting" 3D simulations of architectural environment.

The human been is interaction with the environment. The $3 \mathrm{D}$ simulation for architectural design must have this interactivity.

For millions of people, everywhere, the interaction with the built environment happens into a town. As we know well, in our cities, we enjoy the architectural environment inside a dynamic situation: flows of people and vehicles.

The perception of the architectural environment is always from a dynamic point of view. "Since Brunelleschi's introduction of perspective in the architecture of the 15th century, the relationship between the subject and the object of architecture has been based primarily in the optical, how the subject comes to understand, through sight, his or her experience of the building". (Eisenman, 2002).

Really, can we enjoy the environment without a dynamic point of view? The dynamic point of view is part of our life. The dynamics in the architectural fact, is recently related to the so called "fluid" architecture and "dynamic" architecture. If we analyze, deeply, the phenomena we can understand an emerging topic: the simulation for designing activity should include, more and more, a simulation of dynamic situations.

The architectural fact lives inside a system of flows and spaces: in addition the architecture can be a system of flows and spaces, both built. At today, the space of the simulation should be dynamic, because the design happens, in a new dynamic "space". "Throughout history we have lived in different spaces and architects, using different alphabets, have given them form: informal space, gestural and primitive, pre-Miletus...; the space arterialized by the Greeks and Romans; the sacred and mystic space before Giotto; that perspective space of the Renaissance; the industrial and mechanical, analytical and nonperspective space after Cézanne” (De Kecrkhove 2001).

\subsection{About the theoretical vision...}

The virtual environment is both technical and cultural. The first important factor, from a scientific point of view, is that we are inside the crossroads between some important concepts that combines different research field such: the "spatial analysis", the "scientific visualization", "the flows generation"... we think that, in the future, the designers will use, more and more, these concepts in order to design interactively large size built environment. In fact, the architectural design - before - and the architectural fact - after - both happen in a sort of "Spatial Analysis System".

The way is open to offer to this "system", knowledge, cognitions and theories, that can support the architectural design work, for every application and scale (extended the current use of the archaeological and territorial applications). In this system is possible to include and to develop some current concepts and operative strategies about "spacing", and spatial weaving.

The second important factor is the upgrading meaning of interaction as the catalyzing element of the current architectural research and development activity. Using the new way of the interaction, designers are developing new design theory and practices (movements, paths, metaphors, systems .... to be firstly navigated and then built). The current vanguard of architects try to conceive and built architectural opera able to change, in real time, according with the needs to the people/users: the next architecture will be "in movement". Probably we will use dynamic simulation to understand the simulation of the architectural fact, as well.

Anyway, because the architecture is part of this system of connections, that system has a big influence (before) of the normal way to operate by the architectural design actors and (after) of that architecture, built inside a existing system of connections, links, ...flows.

\section{FUTURE WORK}

As we know, the design activity becomes every day more specialised and complex. At today the meaning of words like "design", "conception", "project", includes various concepts 
mixed together: some of them, are common to the different approaches: cultures, techniques, attitudes, uses, normal ways of thinking, ... we can affirm that the 3D simulations theories, techniques and devices become, every day, a necessary part of this common background ... and not only for the design process, but for the design conception itself (Fiamma P. 2003). The virtual simulations can push the designers to work on a powerful and stimulating design scenario, if this scenario is built and understood like a "system" .

To obtain that goal, we should improve the research for including in the virtual dimension of the design simulations, the different techniques, combined in a sort of "holistic" approach. From a architectural design theory point of view, what is important is what can happen inside the "space" between the use of the virtual reconstruction and the human cognition: this space is virtual, but mental too.

The overlap between different disciplines offers the opportunity to investigate common aspects in order to obtain results that can offer advantages for the single discipline. Assume data, elaborate data, use data for new applications (like architectural design) is a process that goes across several disciplines, that can shape this process itself, through an holistic way.

From architectural design point of view, the data about the building should be converted in data for design as well as to realize a knowledge preservation.

The possibility to have a $3 \mathrm{D}$ laser scanning acquisition, i.e., is at today more powerful with respect to use automatically these data for architectural design. We know the problem about the interpretation of the data and about the possibility to use these data, automatically, changing actors during the process.

Because the environment of the simulation is the virtual reality, the main focus is to develop "tools' of that dimension... but if we analyse, deeply, the state of the art, we understand that there are always a lot of tools, in the different research field...the only problem is that several digital tools are not collaborative each other...

We will to realize a system that can combine the scanner acquisition with the building information modelling B.I.M. procedure, in order to have object oriented data acquisition of technical components for architectural construction, and we will to include this object oriented data in our large size architectural simulation.

The main focus will be how to introduce in the processing data some ontology that can help to realize a constructive hierarchy in the architectural environment.

We figure out a holistic approach to the problem that can stimulate at involve scanning, software programming and architectural designing.

The next step is to make the virtual space of our simulations, more and more accurate, integrated, interactive and dynamic, like not only the reality, but like our human mental space too.

\section{REFERENCES}

References from Books:

De Kerckhove, D. 2001. The Architecture of Intelligence. Birkhauser, Basel, Switzerland.

Lynn G., 1998. Animate form. Princeton University Press, New York, USA.

Schwarzer M., 2004. Zoomscape: architecture in motion and media. Princeton Architectural Press, USA.
Schumacher M., Schaeffer O., Vogt M.-M., 2010. Move: Architecture in Motion - Dynamic Components and Elements. Birkhauser, Basel, Switzerland.

Tschumi B., 1997. Architecture In/of Motion. NAI Publishers, Rotterdam, Holland.

Vidler A., 2000. Warped Space: Art, Architecture, and Anxiety in Modern Culture, MIT Press.

Wagter R., Van den Berg M., Luijpers J., Van Steenbergen M., 2005. Dynamic Architecture: How to Make it Work. John Wiley \& Sons, Hoboken, New Jersey, USA.

References from Other Literature:

Borgeat, L., Godin G., Blais F., Beraldin J-A., Massicotte P., and Poirier G., 2007. Visualizing and analyzing large and detailed 3D dataset. In: The International Archives of Photogrammetry, Remote Sensing and Spatial Information Sciences, ETH Zurich, Switzerland, Vol. XXXVI-5/W47

Seipel, S., Forsberg A.K., 2007. A collaborative visualization environment for natural interaction with I architectural content. In: The International Archives of Photogrammetry, Remote Sensing and Spatial Information Sciences, ETH Zurich, Switzerland, Vol. XXXVI-5/W47

\section{References from websites:}

Eisenman, P., 2002. "Radical Passivity", report on Arch'it Eventi, Roma, Italy.

http://architettura.it/eventi/20060323/index_en.htm

Saggio, A., 2002. Other Challenges. Symposium Designing And Manufacturing Architecture in Digital Age. University of Pennsylvania, USA.

http://www.arc1.uniroma1.it/saggio/conferenze/Phila/Phila.html

Sjarifudin, F. U., Wizaka W., "Collaborative 3D Programming of Space and Event. Conceptual Design tool using Game Engine and Event Pictogram", report on ICSD.

http://www.icsid.org/education/education/articles1088.htm 